BMJ Open

Diabetes

Research

\& Care

\section{Hypoglycemia in patient with type 2 diabetes treated with insulin: it can happen}

To cite: Heller SR, Peyrot M, Oates SK, et al. Hypoglycemia in patient with type 2 diabetes treated with insulin: it can happen. BMJ Open Diab Res Care 2020;8:e001194. doi:10.1136/ bmjdrc-2020-001194

Received 13 January 2020 Revised 29 April 2020 Accepted 9 May 2020
Check for updates

(C) Author(s) (or their employer(s)) 2020. Re-use permitted under CC BY-NC. No commercial re-use. See rights and permissions. Published by BMJ.

${ }^{1}$ Endocrinology \& Metabolism, University of Sheffield,

Sheffield, UK

${ }^{2}$ Sociology, Loyola University Maryland, Baltimore, Maryland, USA

${ }^{3}$ Endocrinology \& Metabolism, Indiana University Health Arnett Hospital, Lafayette, Indiana, USA

${ }^{4}$ Medical Development, Lilly USA, Indianapolis, Indiana, USA

Correspondence to

April D Taylor:

taylor_april_dawn@lilly.com

\section{ABSTRACT}

There are many misconceptions about the prevalence and effects of hypoglycemia in people with type 2 diabetes (T2D), including hypoglycemia does not occur or does not have adverse consequences in T2D. This narrative review aims to help dispel these myths. Around $25 \%$ of people with $\mathrm{T} 2 \mathrm{D}$ taking insulin for $>5$ years were found to have severe hypoglycemic events, which is comparable to the severe hypoglycemia rate in adults with type 1 diabetes (T1D) diagnosed within 5 years. The total number of hypoglycemic events among insulin-treated T2D, including severe hypoglycemia, is as high or higher than among those with T1D. Recent evidence suggests serious consequences of hypoglycemia may, in some respects, be greater in individuals with T2D, particularly regarding effects on the cardiovascular system. Hypoglycemia is generally patient-reported. Issues with hypoglycemia unawareness, limited glucose testing, limited recall, lack of event logging and fear of failure or shaming limits the number of hypoglycemic episodes reported by people with diabetes. Barriers to healthcare provider inquiry and reporting include lack of knowledge regarding the problem's magnitude, competing priorities during patient visits, lack of incentives to report and limitations to documentation systems for adequate reporting. All people with diabetes should be encouraged to discuss their experiences with hypoglycemia without judgment or shame. Glucose targets, testing schedules (blood glucose or continuous glucose monitoring) and treatment plans should be reviewed often and individualized to the minimize risk of hypoglycemia. Finally, people with T2D on insulin should always be encouraged to have oral glucose and rescue medication immediately available.

\section{INTRODUCTION}

The risk of hypoglycemia in people with type 1 diabetes (T1D) is clearly appreciated as a side effect of treatment. It is not as clearly understood in those with type 2 diabetes (T2D) treated with insulin. The risk of hypoglycemia in people with T2D has been considered less important, with the implication that it can often be ignored. Recent evidence contradicts this belief and suggests that serious consequences of hypoglycemia may, in some respects, be greater in individuals with T2D, particularly with regard to its effects on the cardiovascular system. ${ }^{12}$
Furthermore, in older people with T2D who often live alone, comorbidities (eg, cognitive impairment and dementia, impaired renal function, other medications and frailty) can make hypoglycemia far more dangerous and difficult to manage.

Despite several studies addressing this topic, ${ }^{3-15}$ there are still lingering myths about hypoglycemia in people with T2D treated with insulin:

- People with T2D rarely experience hypoglycemia.

- People with T2D with elevated hemoglobin A1c (HbAlc) are not at risk of hypoglycemia.

- People with T2D can have hypoglycemia, but it is not severe due to the body's counter-regulatory responses.

- Hypoglycemia does not result in adverse consequences for people with T2D.

This narrative review aims to dispel these myths by examining the physiology, comorbidities and reported incidence and rates of hypoglycemia in people with T2D. Moreover, we consider why hypoglycemia is often underreported and under-identified by individuals and healthcare providers (HCPs), and how we can improve hypoglycemia detection, prevention and treatment education. Throughout this narrative review, the term 'hypoglycemia' is intended to generally refer to any level of severity; concepts related to 'severe hypoglycemia' are specified as such.

\section{UNDERSTANDING HYPOGLYCEMIA FROM A MANAGEMENT PERSPECTIVE \\ Pathophysiology of hypoglycemia}

In people without diabetes, hypoglycemia is rare since endogenous insulin secretion is inhibited as glucose levels fall below normal. Individuals with diabetes, treated with insulin, sulfonylureas or other insulin secretagogues, are at increased hypoglycemic risk because glucose levels will continue to fall until either insulin boluses or the effect of oral medication 
are cleared. At diagnosis, individuals with diabetes are protected, at least in part, from the glucose-lowering effect of insulin by physiological responses initiated by stress pathways. As blood glucose falls below normal, glucagon is released by $\alpha$ cells probably due to a paracrine response within the islets. Glucagon is a powerful counter-regulatory hormone that defends individuals against hypoglycemia by stimulating release of glucose from the liver through glycogenolysis.

With increased duration of diabetes, in both T1D and advanced T2D, progressive loss of $\beta$ cells prevents paracrine cross-talk between the $\alpha$ and $\beta$ cells leading to impaired glucagon release during hypoglycemia. This increases vulnerability to hypoglycemia. Thus, duration of T1D and, in T2D, the duration of insulin treatment are important predictors of hypoglycemic risk.

Those affected with declining $\beta$-cell function and impaired glucagon responses are still protected from hypoglycemia by activation of the sympathoadrenal nervous system and associated release of circulating epinephrine, but this defense also can become impaired. It is particularly sensitive to antecedent episodes of hypoglycemia, which reset the threshold for epinephrine release to a lower glucose level. One consequence is that the counter-regulatory response is delayed and, as glucose levels fall below $3 \mathrm{mmol} / \mathrm{L}(54 \mathrm{mg} / \mathrm{dL})$, individuals can become cognitively impaired and are at major risk of a severe hypoglycemic episode. This has been described by some as 'hypoglycemia-associated autonomic failure'. However, it is important to note that this condition is not closely related to diabetic autonomic neuropathy, which reflects structural and irreversible damage to the autonomic nervous system. Defective counter-regulatory responses to hypoglycemia as a result of antecedent episodes are functional rather than structural, at least in part. They can be reversed by scrupulous avoidance of hypoglycemia, an important treatment option in those affected in this way.

In summary, these pathophysiological changes, which both diminish symptomatic responses as well as reduce the ability of the body to resist the glucose-lowering effect of insulin, explain why individuals with T1D or long-standing T2D both are vulnerable to hypoglycemia during treatment.

\section{Hypoglycemia prevalence in people with T2D}

In a trial of participants with T2D treated with insulin glargine, hypoglycemia (defined as blood glucose $\leq 3.3 \mathrm{mmol} / \mathrm{L}(60 \mathrm{mg} / \mathrm{dL}))$ was detected in $56.9 \%$ of the participants by open-label, 3-day, blinded continuous glucose monitoring (CGM) as compared with $26.4 \%$ of the participants by their 8-point profile, self-monitored blood glucose. ${ }^{14}$ In a study using 5-day blinded CGM, Gehlaut et alfound that $75 \%$ of participants with T2D were unaware of hypoglycemic episodes (defined as glucose $<3.9 \mathrm{mmol} / \mathrm{L}(70 \mathrm{mg} / \mathrm{dL}))$ detected by CGM. ${ }^{4}$ While best practice is to confirm suspected or CGM-identified hypoglycemia by self-monitored blood glucose, it is clear that much hypoglycemia objectively identified by CGM is not checked when CGM readings are not available in realtime. These studies suggest that the frequency of hypoglycemia in T2D is much higher than previously appreciated by both clinicians or people with T2D themselves.

\section{Insulin treatment duration and hypoglycemia rates}

In a multicenter observational study, UK researchers recruited individuals with either T2D or T1D to participate in a 9-month to 12-month prospective study in which their hypoglycemic burden was measured by questionnaires and CGM. ${ }^{16}$ People with T2D were divided into three treatment groups: insulin for $<2$ years, insulin for $>5$ years and sulfonylureas. The study reported in 2007 that self-reported severe hypoglycemic episodes occurred in about $7 \%$ of the group with T2D who had been taking insulin for $<2$ years, a frequency comparable to the group taking sulfonylureas. The group with T2D taking insulin for over 5 years had a significantly higher risk of severe hypoglycemia, with around $25 \%$ reporting a severe hypoglycemic episode during the study. Thus, between 2 and 5 years of insulin treatment, rates of self-reported severe hypoglycemia in individuals with T2D had tripled.

The UK Hypoglycaemia Study Group also reported that the proportion of patients with T2D taking insulin for over 5 years who reported at least one severe hypoglycemic event was comparable to that of adults with T1D diagnosed within the last 5 years, and lower than adults with T1D for $>15$ years. ${ }^{16}$ Although reported perperson-year rates of hypoglycemia are higher for T1D than insulin-treated T2D ${ }^{5}$ there are many more people with insulin-treated T2D, ${ }^{17} 18$ resulting in more events of hypoglycemia (especially severe hypoglycemia resulting in hospitalization) among the insulin-treated T2D population. $^{19}$

\section{Consequences of hypoglycemia}

It is beyond the scope of this review to give a detailed account of the consequences of hypoglycemic episodes, but recent trials have raised the possibility that, in addition to the well-recognized effects of hypoglycemia on the central nervous system, hypoglycemia may also have adverse consequences on the cardiovascular system. ${ }^{12}$ Considerable evidence, including both clinical trials and observational studies, has demonstrated a consistent association between hypoglycemia and adverse cardiovascular outcomes. The current debate is focused on whether hypoglycemia is a risk factor, implying causality or merely a risk marker such that hypoglycemia is more common in vulnerable individuals due to frailty and comorbidity. In the absence of randomized controlled trials (RCTs), which are neither feasible nor ethical, it is challenging to prove this either way, although many believe that both mechanisms are contributing. However, the strength and consistency of the association reinforces the importance of choosing treatment which minimizes the risk of hypoglycemia, particularly in vulnerable individuals. 
Evidence also suggests a two-way relationship between hypoglycemia and impaired cognitive function and dementia. Significantly impaired cognition reduces the ability to self-manage diabetes, increasing the risk of hypoglycemia, while repeated hypoglycemia can lead to neurodegeneration, contributing to dementia and cognitive decline. ${ }^{20}$ Non-severe hypoglycemia has also been found to decrease cognitive function during an insulininduced episode in people with T2D. ${ }^{21}$

\section{Hypoglycemia rates reported in clinical trials}

The belief that hypoglycemia is less of a problem in T2D may stem, in part, from low rates reported for RCTs of individuals with newly diagnosed T2D. In the UK Prospective Diabetes Study (UKPDS), individuals with newly diagnosed T2D were assigned to either intensive treatment with insulin or a sulfonylurea, or conventional treatment. The UKPDS results published in 1998 showed that tighter glucose control reduced the incidence of microvascular complications, but increased the frequency of hypoglycemic episodes $(0.7 \%$ per year with conventional treatment vs $1.8 \%$ per year with insulin).$^{22}$ For individuals who remained on the intensive treatment for 6 years, UKPDS researchers reported a frequency of $2.5 \%$ per year of what they described as 'substantive hypoglycemic episodes' and concluded that hypoglycemia was unlikely to be a barrier to achieving tight glucose targets using insulin or sulfonylureas. ${ }^{13}$ In the randomized controlled Diabetes Control and Complications Trial (DCCT) results published in 1997, benefits of intensive treatment were observed despite a marked increase in the rate of severe hypoglycemia. ${ }^{23}$

A newly diagnosed person with T2D is not representative of the typical insulin-taking person with T2D since, as described above, counter-regulatory defenses that confer protection against severe episodes are intact at diagnosis. At the time of the UKPDS and DCCT trials, it was perhaps underappreciated that conclusions based on a study in newly diagnosed individuals are, therefore, not a reliable guide to the risks of hypoglycemia in individuals with T2D on insulin. The need for insulin treatment in people with T2D reflects a progressive failure of $\beta$-cell secretion of endogenous insulin. Newly diagnosed individuals with T2D, if prescribed insulin, will require significantly less exogenous insulin and have a significantly better counterregulatory response compared with individuals with advanced diabetes. ${ }^{24}$ However, insulin is not a first-line treatment recommendation for newly diagnosed individuals with $\mathrm{T}_{2} \mathrm{D}^{25}$ and is not frequently prescribed in those individuals. ${ }^{24}{ }^{26}$ On average, insulin was found to be initiated in people with T2D $>7$ years after initiation of oral antidiabetic agent(s) even despite elevated HbAlc levels. ${ }^{26}$ The insulin resistance of T2D may also reduce hypoglycemic risk. As endogenous insulin diminishes, the physiological counter-regulatory defense also decreases, as described in Pathophysiology of hypoglycemia. ${ }^{24}$

Finally, prior to a recent International Hypoglycaemia Study Group position statement, ${ }^{27}$ no consistent, standardized reporting of levels of hypoglycemia were used in clinical trials. Each clinical trial protocol defined the glucose levels used to determine hypoglycemic events, which contributed to the misconceptions about the prevalence and effects of hypoglycemia in people with T2D on insulin. The position statement provided guidelines for the standardization of reporting levels for hypoglycemia, with clinically important hypoglycemia (level 2) defined as glucose level $<3 \mathrm{mmol} / \mathrm{L}$ (54mg/dL) and severe hypoglycemia (level 3) denoted by severe cognitive impairment requiring external assistance for recovery. ${ }^{27}$ In addition, time-in-range goals have been defined as metrics for CGM. ${ }^{28}$

\section{Clinical study design impacts hypoglycemia rate}

We need to draw on studies that are population-based to provide an accurate measure of hypoglycemic risk in those with diabetes. A study by Donnelly et al published in 2005 is one of the few that measured rates of hypoglycemia in a random sample of individuals with insulin-treated diabetes in a single city. This study measured rates of hypoglycemia over a 1-month period. They reported that participants with T2D treated with insulin had about 16 hypoglycemic events per person per year, with the equivalent of 0.35 severe events per person per year. Significant predictors of hypoglycemia for people with T2D were a history of hypoglycemia and duration of insulin treatment. Other important risk factors include declining renal function, increasing age, impaired cognitive function and dementia.

More recent data from a global, multicenter study indicate that the diabetes community may have underestimated the frequency of both severe and non-severe episodes of hypoglycemia. The Hypoglycemia Assessment Tool (HAT) study published in 2016 involved over 27000 insulintreated adults with T1D or T2D from 24 countries. ${ }^{5}$ Study centers were located in both high-income and low-income and middle-income countries and data were recorded both retrospectively and prospectively. In those with T2D, the overall rate of severe hypoglycemia was around 2.5 events per person per year, which is about seven times higher than the rate reported by Donnelly et al. In addition, there was no association between the rate of hypoglycemia and $\mathrm{HbAlc}$ in the HAT study, an observation also reported in other studies. ${ }^{89}$ It is likely that the method of recording, which involved online surveys, may have introduced a degree of selection bias; however, the high rates of hypoglycemia are of considerable concern despite these study limitations.

\section{HYPOGLYCEMIA REPORTING}

\section{Patient under-identifying and under-reporting}

As described above, hypoglycemia does occur in people with T2D; however, both recognition and reporting are limited. Effective mitigation measures can only be implemented by the individual, the healthcare team and the payer once the incidence and causes of hypoglycemia are identified.

Although there are many potential sources of information about the frequency and severity of hypoglycemic episodes in the general diabetes population, the individual is the primary source of information. 
Unfortunately, people with diabetes often do not report hypoglycemic episodes to their HCPs. In one study, $67 \%$ of people with T1D and $43 \%-53 \%$ of those with T2D rarely reported hypoglycemia to their HCPs. ${ }^{12}$ In another study, $60 \%$ (T1D) and $46 \%$ (T2D) rarely or never report hypoglycemia to their HCPs. ${ }^{10}$ Given the central role of individuals in making HCPs aware of hypoglycemia, it is important to understand the process that generates opportunities for sharing and the factors affecting the responses to these opportunities. Below we describe this chain of events: (1) identifying/recognizing hypoglycemic events, (2) involving HCPs in treating hypoglycemic events, (3) recalling hypoglycemic episodes and (4) sharing information about hypoglycemic episodes during HCP visits.

\section{Identifying/Recognizing hypoglycemic events}

The first item in the chain is identifying/recognizing a hypoglycemic event. There is general understanding that individuals with insulin-treated diabetes can develop impaired hypoglycemic awareness or hypoglycemic unawareness. This results in increased numbers of asymptomatic, non-severe hypoglycemic events, ${ }^{7}$ which contributes to a higher incidence of severe episodes when corrective action is not taken until hypoglycemic symptoms become severe. ${ }^{3031}$ People with diabetes may not collect a glucose data point when experiencing hypoglycemia because they are occupied treating the low blood glucose. This limits any quantitative measure of the episode or a record for future analysis. Non-severe episodes can also be misinterpreted by people with diabetes and/or family members as a decrease in cognition, particularly in the elderly and not recognized as hypoglycemia. Moreover, one-third or more of people with T1D and T2D define/ identify hypoglycemia by the presence of symptoms. ${ }^{11}$ Thus, only a portion of hypoglycemic episodes are available for sharing.

\section{Involving healthcare providers in treating hypoglycemic events}

The second item in the chain is involving HCPs in treating hypoglycemic events. HCPs often are not informed when hypoglycemia occurs, whether severe or non-severe; only 26\% (T1D) and 33\% (T2D) of people with diabetes sought medical care for hypoglycemic events and only 24\% (T1D) and 31\% (T2D) consulted with their own physician/nurse. ${ }^{11}$ The opportunity for treatment support is missed, and sharing information with the individual's HCP requires a separate action following the event, which creates additional barriers to sharing.

\section{Recalling hypoglycemic episodes}

The third item in the chain is recalling hypoglycemic episodes. While almost $90 \%$ of individuals with a documented, severe hypoglycemic episode within the last year recalled that they had such an episode, those experiencing frequent episodes 'had incomplete recall, resulting in a $15 \%$ underestimation of the overall rate'. ${ }^{31}$ In a research study of mild hypoglycemia, recall was low over a 4-week period $(48 \% \text { for T1D and } 75 \% \text { for T2D })^{11}$; given the 3-month to 4-month interval for routine diabetes care visits (even among conscientious individuals), it is likely that recall would be even lower during such visits.

\section{Sharing information about hypoglycemic episodes during every} healthcare provider visit

The fourth item in the chain is sharing information about hypoglycemic episodes during HCP visits. The loss of information due to under-recognition and incomplete recall limits the information available for sharing. But even when individuals have the relevant information in hand, there are reasons they may not raise the subject of hypoglycemia. The needs of the individual and provider are not always aligned due to limitations in visit times and competing agendas. This can lead to less frequent inquiries about hypoglycemia.

Beyond competing priorities, there may be specific disincentives in reporting hypoglycemic experience. For example, when EU legislation was implemented allowing withdrawal of a driver's license for repeated, severe hypoglycemic episodes, the number of such episodes recorded in medical records during the year following enactment dropped by $55 \%{ }^{32}$ The conclusion that this was a result of reduced reporting rather than a reduced episode rate was supported by an anonymous survey of the cohort of 309 adults with T1D, which demonstrated that the medical record episodes were $70 \%$ lower than reported in the survey. ${ }^{32}$ Finally, HCPs sometimes express negative judgments to people with diabetes regarding less than desired treatment outcomes, which creates a barrier to sharing information about hypoglycemia. People with diabetes express hesitation to report episodes of hypoglycemia due to fear of HCP disappointment, as well as feelings of guilt and shame. ${ }^{33}$

\section{Healthcare provider under-identifying and under-reporting}

HCPs often under-identify and under-report episodes of hypoglycemia. The combination of HCP expectations/ beliefs regarding hypoglycemia, limited time during clinical visits, variable degrees of provider-patient trust and competing agendas may all limit the HCP's ability to identify or report hypoglycemia.

\section{Expectations based on published rates of hypoglycemia}

HCPs look to the literature for rates of hypoglycemia, but the available information can be confusing. For example, Elliot et al published a review of hypoglycemia rates from 19 RCTs and 11 real-world data studies. ${ }^{34}$ We might expect that the higher level of oversight, more frequent data collection, and increased expectations of individuals enrolled in RCTs would increase the detection rate of hypoglycemia, but intensified patient follow-up and engagement decreases the actual incidence of hypoglycemia. Furthermore, individuals with an increased risk of hypoglycemia, including those with impaired awareness, are frequently excluded from trials. Thus, rates of hypoglycemia are consistently higher in real-world data 
studies compared with RCTs. This suggests that published rates of hypoglycemia from clinical trials underestimate the actual rate of hypoglycemia in the population, which may skew expectations of those who care for people with diabetes.

\section{Patient history review}

During routine visits, HCPs are often asked to record considerable clinical information and may be documenting items such as date of the last eye examination or name of the podiatrist providing foot care. Patients report that their physicians frequently fail to inquire about hypoglycemia. In one study, $10 \%$ (T1D) and 13\% (T2D) of people with diabetes reported that they were not asked about their hypoglycemia during consultations. ${ }^{10}$ Trying to cover all the required topics may be one reason that hypoglycemia is under-identified. This represents an HCP opportunity for increasing identification and reporting. Another lies with inquiry of the family or friends who accompany the person with diabetes. Asking for input from family members increases reporting of severe hypoglycemia and helps HCPs understand the context and impact on the family and others. ${ }^{35}$

Hypoglycemia rates may not be reported to HCPs by people with diabetes because of the above-mentioned negative feedback. In office practices, the support staff may be the ones who inquire about hypoglycemia rates. One way to improve communication with the person with diabetes and the family is to normalize this question about hypoglycemia by asking every time and routinely using technology to capture glucose values. HCPs should at the very least inquire about low glucose values or awareness of hypoglycemia, but conversations regarding all aspects of diabetes care should be non-judgmental.

\section{Quality measures and reporting tools}

Consistent, clear documentation of frequency, timing and causes of hypoglycemia for people with T2D on insulin allows HCPs, health systems and payers to understand the magnitude of hypoglycemia. Use of diabetesout-of-control codes versus use of hypoglycemia codes buries the problem within the documentation systems. Standardization of reporting language in both trials and clinical settings is key to a clear understanding of the incidence/prevalence and severity of hypoglycemic events.

The rate of hypoglycemia has not been a required field for ambulatory quality reporting measures in the USA, so providers and healthcare systems are not incentivized to document it. The focus for quality measures assessed by the National Committee for Quality Assurance has been on HbA1c testing and control, retinal eye exams, addressing nephropathy and blood pressure control. ${ }^{36}$

A workgroup convened by the American Diabetes Association and The Endocrine Society designed several tools to optimize collection of information around hypoglycemia and create a framework for addressing the problem. ${ }^{37}$ The workgroup created a one-page questionnaire for individuals and another checklist for providers to review at every visit.

\section{HYPOGLYCEMIA DETECTION \\ Patient monitoring \\ Blood glucose monitoring}

Home blood glucose monitoring is facilitated by easyto-use portable devices. Some individuals with T2D test often, while some never test. ${ }^{38}$ The number and timing of prescribed glucose tests per day should be individualized to the needs of each person with diabetes. ${ }^{39}$ However, payers often restrict glucose monitor models and/or strip allowance and frequently change coverage yearly. Finally, out-of-pocket cost and the pain of the finger sticks also limit testing.

No consistency is found in the literature for the recommended number of blood glucose tests per day for people with T2D on insulin. The American Diabetes Association Standards of Care acknowledge the importance of blood glucose testing for those with T2D and recommends an individualized approach to the frequency of testing. ${ }^{39}$ Rama Chandran et al found that four to five blood glucose tests per day was enough to predict hypoglycemia comparable to CGM. ${ }^{29}$ Although often resisted by people with diabetes, prediction and prevention of hypoglycemia is an excellent reason to suggest multiple daily blood glucose testing for people with T2D treated with insulin. ${ }^{29} 40$ A large observational study of patients in Italy found that $26.4 \%$ of participants tested blood glucose less than once a day and the incidence of hypoglycemia was inversely correlated with the number of daily blood glucose tests performed. ${ }^{41}$ In addition to scheduled blood glucose tests, blood glucose also should be tested when low glucose is suspected, either before or during treatment, and retested every $15 \mathrm{~min}$ after treatment until resolution. ${ }^{39}$

In the clinical setting, people with diabetes are encouraged to bring their glucose monitors with them for data download. Some individuals bring their monitors, while others leave them at home. Some people with diabetes do not know how to interpret their glucose levels or how to improve their control. ${ }^{42}$ Digital data reports or paper logbook review at each visit allows for conversations between the person with diabetes and the healthcare team about glucose patterns (including hypoglycemic episodes), areas of success and areas of needed improvement.

If a person with diabetes receives limited to no feedback from the HCP about glucose levels, some will not consistently test. Inattention by the HCP to the glucose data reports can lead to lower individual motivation to test, record or report. ${ }^{43}$

\section{Continuous glucose monitoring}

CGM or flash glucose monitoring (FGM) may be preferred to blood glucose monitoring. ${ }^{94}$ Personal monitors provide glucose data to the person with diabetes 
in real-time and all models have trending arrows that give insight into glucose trends. For many people with diabetes (whether T1D or T2D), asking them to perform a blood glucose fingerstick test multiple times per day can be an unrealistic expectation. The use of CGM or FGM provides multiple glucose measures throughout each 24 hours period without frequent fingersticks. However, cost and coverage are limiting factors for widespread use in many countries.

Clinicians can intermittently use a one-time blinded CGM for a 6-day to 14-day data collection period (depending on the model) to assess glucose patterns. ${ }^{45-48}$ As stated above, the use of CGM in research studies has demonstrated that people with T2D experience a higher than previously appreciated number of clinically relevant hypoglycemia episodes. Use of the CGM was shown to lead to treatment modification in $64.4 \%$ of CGM users (decreased drug dose, decreased drug frequency, change in drug administration timing, stopping drug completely or adjusting glucose targets). ${ }^{4}$

\section{Tracking and logging}

Electronic logbooks have entered the market and allow for more robust data capturing. Most blood glucose and CGM monitors allow the user and the HCP to connect to a mobile application or a professional data visualization program to review and share data. However, not all the monitors and/or mobile applications track the level of detail required to fully understand the cause, severity of symptoms or treatments employed regarding a hypoglycemic episode. Until these meters or applications provide opportunity to capture these details, for some individuals, it may warrant keeping a paper log that is reviewed at each clinic visit. In elderly people, who are most at risk, relatives often need to be interviewed to provide the details of hypoglycemic episodes and causes and should attend visits whenever possible.

\section{HYPOGLYCEMIA PREVENTION AND TREATMENT EDUCATION Prevention}

Prevention of hypoglycemia remains problematic as demonstrated by the high rates observed in various studies. The first step to prevention is acknowledgment of the risk of hypoglycemia in the T2D population treated with insulin. Next, avoidance of excess glucose fluctuations should be an important consideration for reducing the risk of hypoglycemia in T2D. Consideration should be made for therapies that have a lower risk of glucose variability. ${ }^{29}$ The overall goal is not simply to lower the $\mathrm{HbAlc}$, but to create stable glucoses in a range to decrease complications and reduce variability. ${ }^{6950}$ When a CGM or FGM is used, review of the time graph and glucose profile with the patient allows for open discussions about incidence, timing and causes of hypoglycemia. Most importantly, ongoing review of the data accompanied by clear goals and an action plan should occur at every visit.

\section{Treatment and prevention education}

It is important to inquire about all levels of hypoglycemia at each visit with an open, shame-free discussion. The HCP and patient should collaborate to troubleshoot causes and create solutions. ${ }^{37}$ The Hypoglycemia Work Group provides a Hypoglycemia Patient Questionnaire and a Hypoglycemia Provider Checklist to guide these discussions. ${ }^{37}$ The International Hypoglycaemia Study Group has several resources available to support people with diabetes as well. ${ }^{51}$ We also advocate using patient portal communication to adjust therapies between visits as needed.

The frequency of severe hypoglycemia in T2D can be significantly reduced after participation in structured interventions that include patient education. ${ }^{52}$ We advocate the training of providers (including primary care providers), staff, people with diabetes and their families on the creation of an action plan for timely and appropriate treatment of hypoglycemia. The International Hypoglycaemia Study Group provides several resources to assist HCPs in the understanding of hypoglycemia causes and treatments. ${ }^{51}$ The use of oral glucose is preferred as firstline treatment when the person with diabetes is coherent and able to swallow. For anyone treated with insulin, a rescue treatment plan needs to be in place should the person with diabetes become unable to self-treat. ${ }^{39}$

\section{CONCLUSION}

Hypoglycemia has been found to contribute to cardiovascular and central nervous system harm in people with T2D. Hypoglycemia is a risk for people with T2D being treated with insulin, with reported rates of severe hypoglycemia around 2.5 events per person per year. ${ }^{5}$ However, unless it is severe, hypoglycemia is primarily a patient-reported event and is not fully apparent in both recent trial design and in patient-provider interactions for people with T2D. An improved understanding of the barriers to accurate reporting would assist the diabetes community in estimating the frequency and severity of hypoglycemic episodes in people with T2D. Consistent clinical study designs that use standardized hypoglycemia reporting levels and definitions of time-in-range goals will contribute to the accurate assessment of hypoglycemic risk in people with T2D.

Barriers to the HCP, including competing priorities during patient visits, lack of incentives to report and limitations to documentation systems, should be addressed to ensure hypoglycemia is assessed and recorded in all people with T2D on insulin. The collaboration between HCPs and people with T2D is a key component of hypoglycemia prevention, monitoring and treatment. Addressing issues surrounding hypoglycemia awareness, glucose testing, episode recall and event logging can improve reporting by people with diabetes. HCP feedback helps motivate people with diabetes to test, record and report glucose levels. All people with diabetes should 
be encouraged to discuss their experiences with hypoglycemia without judgment or shame.

HCPs (including primary care providers), clinical staff, people with diabetes and their families should be encouraged to work together toward creating an action plan for timely and appropriate treatment of hypoglycemia. The ongoing review of glucose level data should occur at every clinic visit. Glucose targets, testing schedule and the treatment plan should be reviewed often and individualized to minimize the risk of hypoglycemia. Finally, people with T2D on insulin should always be encouraged to have oral glucose and a rescue medication immediately available.

Acknowledgements The authors would like to thank Amanda Hodgson, PhD, of Covance (Madison, Wisconsin, USA) for providing writing and editing assistance.

Contributors SRH made substantial contributions to the data interpretation, drafting and critical revision of this work. MP and SKO made substantial contributions to the conception, design, drafting and critical revision of this work. ADT made substantial contributions to the conception, design, data interpretation, drafting and critical revision of this work.

Funding This work was funded by Eli Lilly and Company.

Competing interests SRH has served as a consultant and on advisory boards for Eli Lilly and Company, Novo Nordisk, Lexicon and Zealand Pharma. SRH reports personal fees from Novo Nordisk and fees for lectures from AstraZeneca. MP reports personal fees and non-financial support from Eli Lilly and Company, Calibra, Novo Nordisk, Lifescan and Valeritas. ADT is an employee of Lilly USA, and a minor stockholder of Eli Lilly and Company.

\section{Patient consent for publication Not required.}

Provenance and peer review Not commissioned; externally peer reviewed.

Data availability statement No data are available.

Open access This is an open access article distributed in accordance with the Creative Commons Attribution Non Commercial (CC BY-NC 4.0) license, which permits others to distribute, remix, adapt, build upon this work non-commercially, and license their derivative works on different terms, provided the original work is properly cited, appropriate credit is given, any changes made indicated, and the use is non-commercial. See: http://creativecommons.org/licenses/by-nc/4.0/.

\section{ORCID ID}

April D Taylor http://orcid.org/0000-0002-6725-1942

\section{REFERENCES}

1 International Hypoglycaemia Study Group. Hypoglycaemia, cardiovascular disease, and mortality in diabetes: epidemiology, pathogenesis, and management. Lancet Diabetes Endocrinol 2019;7:385-96.

2 Ismail-Beigi F, Craven T, Banerji MA, et al. Effect of intensive treatment of hyperglycaemia on microvascular outcomes in type 2 diabetes: an analysis of the Accord randomised trial. Lancet 2010;376:419-30.

3 Donnelly LA, Morris AD, Frier BM, et al. Frequency and predictors of hypoglycaemia in type 1 and insulin-treated type 2 diabetes: a population-based study. Diabet Med 2005;22:749-55.

4 Gehlaut RR, Dogbey GY, Schwartz FL, et al. Hypoglycemia in Type 2 Diabetes--More Common Than You Think: A Continuous Glucose Monitoring Study. J Diabetes Sci Technol 2015;9:999-1005.

5 Khunti K, Alsifri S, Aronson R, et al. Rates and predictors of hypoglycaemia in 27585 people from 24 countries with insulintreated type 1 and type 2 diabetes: the global HAT study. Diabetes Obes Metab 2016:18:907-15.

6 Klimontov VV, Myakina NE. Glucose variability indices predict the episodes of nocturnal hypoglycemia in elderly type 2 diabetic patients treated with insulin. Diabetes Metab Syndr 2017;11:119-24.

7 Kulzer B, Seitz L, Kern W. Real-World patient-reported rates of non-severe hypoglycaemic events in Germany. Exp Clin Endocrinol Diabetes 2014;122:167-72.

8 Lipska KJ, Warton EM, Huang ES, et al. Hba1C and risk of severe hypoglycemia in type 2 diabetes: the diabetes and aging study. Diabetes Care 2013;36:3535-42.
9 Mangrola D, Cox C, Furman AS, et al. Self blood glucose monitoring underestimates hyperglycemia and hypoglycemia as compared to continuous glucose monitoring in type 1 and type 2 diabetes. Endocr Pract 2018;24:47-52.

10 Peene B, D'Hooge D, Vandebrouck T, et al. Patient-Reported frequency, awareness and patient-physician communication of hypoglycaemia in Belgium. Acta Clin Belg 2014;69:439-45.

11 Pongrac Barlovič D, Zavratnik A, Skvarča A, et al. Self-Reported hypoglycaemia in patients treated with insulin: a large Slovenian retrospectively-prospective study. Zdr Varst 2017;56:244-50.

12 Weitgasser R, Lopes S. Self-Reported frequency and impact of hypoglycaemic events in insulin-treated diabetic patients in Austria. Wien Klin Wochenschr 2015;127:36-44.

13 Wright AD, Cull CA, Macleod KM, et al. Hypoglycemia in type 2 diabetic patients randomized to and maintained on monotherapy with diet, sulfonylurea, metformin, or insulin for 6 years from diagnosis: UKPDS73. J Diabetes Complications 2006;20:395-401.

14 Zick R, Petersen B, Richter M, et al. Comparison of continuous blood glucose measurement with conventional documentation of hypoglycemia in patients with type 2 diabetes on multiple daily insulin injection therapy. Diabetes Technol Ther 2007;9:483-92.

15 NCD Risk Factor Collaboration (NCD-RisC). Worldwide trends in diabetes since 1980: a pooled analysis of 751 population-based studies with 4.4 million participants. Lancet 2016;387:1513-30.

16 UK Hypoglycaemia Study Group. Risk of hypoglycaemia in types 1 and 2 diabetes: effects of treatment modalities and their duration. Diabetologia 2007;50:1140-7.

17 Bullard KM, Cowie CC, Lessem SE, et al. Prevalence of diagnosed diabetes in adults by diabetes type - United States, 2016. MMWR Morb Mortal Wkly Rep 2018;67:359-61.

18 Sharma M, Nazareth I, Petersen I. Trends in incidence, prevalence and prescribing in type 2 diabetes mellitus between 2000 and 2013 in primary care: a retrospective cohort study. BMJ Open 2016;6:e010210.

19 Zhong VW, Juhaeri J, Cole SR, et al. Incidence and trends in hypoglycemia hospitalization in adults with type 1 and type 2 diabetes in England, 1998-2013: a retrospective cohort study. Diabetes Care 2017;40:1651-60.

20 Shinohara M, Sato N. Bidirectional interactions between diabetes and Alzheimer's disease. Neurochem Int 2017;108:296-302.

21 Nilsson M, Jensen N, Gejl M, et al. Experimental non-severe hypoglycaemia substantially impairs cognitive function in type 2 diabetes: a randomised crossover trial. Diabetologia 2019;62:1948-58.

22 Intensive blood-glucose control with sulphonylureas or insulin compared with conventional treatment and risk of complications in patients with type 2 diabetes (UKPDS 33). UK prospective diabetes study (UKPDS) group. Lancet 1998;352:837-53.

23 The Diabetes Control and Complications Trial Research Group. Hypoglycemia in the diabetes control and complications trial. Diabetes 1997;46:271-86.

24 Segel SA, Paramore DS, Cryer PE. Hypoglycemia-Associated autonomic failure in advanced type 2 diabetes. Diabetes 2002;51:724-33.

25 American Diabetes Association. 9. Pharmacologic Approaches to Glycemic Treatment: Standards of Medical Care in Diabetes-2019. Diabetes Care 2019;42:S90-102.

26 Khunti K, Wolden ML, Thorsted BL, et al. Clinical inertia in people with type 2 diabetes: a retrospective cohort study of more than 80,000 people. Diabetes Care 2013;36:3411-7.

27 International Hypoglycaemia Study Group. Glucose concentrations of less than $3.0 \mathrm{mmol} / \mathrm{L}(54 \mathrm{mg} / \mathrm{dL})$ should be reported in clinical trials: a joint position statement of the American diabetes association and the European association for the study of diabetes. Diabetes Care 2017;40:155-7.

28 Battelino T, Danne T, Bergenstal RM, et al. Clinical targets for continuous glucose monitoring data interpretation: recommendations from the International consensus on time in range. Diabetes Care 2019;42:1593-603.

29 Rama Chandran S, Tay WL, Lye WK, et al. Beyond HbA1c: comparing glycemic variability and glycemic indices in predicting hypoglycemia in type 1 and type 2 diabetes. Diabetes Technol Ther 2018;20:353-62.

30 Hendrieckx C, Hagger V, Jenkins A, et al. Severe hypoglycemia, impaired awareness of hypoglycemia, and self-monitoring in adults with type 1 diabetes: results from diabetes MILES-Australia. $J$ Diabetes Complications 2017;31:577-82.

31 Pedersen-Bjergaard U, Pramming S, Thorsteinsson B. Recall of severe hypoglycaemia and self-estimated state of awareness in type 1 diabetes. Diabetes Metab Res Rev 2003;19:232-40. 
32 Pedersen-Bjergaard U, Færch L, Allingbjerg M-L, et al. The influence of new European Union driver's license legislation on reporting of severe hypoglycemia by patients with type 1 diabetes. Diabetes Care 2015;38:29-33.

33 Archer A. Shame and diabetes self-management. Practical Diabetes 2014;31:102-6.

34 Elliott L, Fidler C, Ditchfield A, et al. Hypoglycemia event rates: a comparison between real-world data and randomized controlled trial populations in insulin-treated diabetes. Diabetes Ther 2016;7:45-60.

35 Pedersen-Bjergaard U, Thorsteinsson B. Reporting severe hypoglycemia in type 1 diabetes: facts and pitfalls. Curr Diab Rep 2017; $17: 131$

36 National Commitee for Quality Assurance. Healthcare effectiveness data and information set (HEDIS) measures for comprehensive diabetes care, 2019. Available: https://www.ncqa.org/hedis/ measures/comprehensive-diabetes-care/ [Accessed 16 Dec 2019].

37 Seaquist ER, Anderson J, Childs B, et al. Hypoglycemia and diabetes: a report of a Workgroup of the American diabetes association and the endocrine Society. Diabetes Care 2013;36:1384-95.

38 Harris MI, National Health and Nutrition Examination Survey (NHANES III). Frequency of blood glucose monitoring in relation to glycemic control in patients with type 2 diabetes. Diabetes Care 2001;24:979-82

39 American Diabetes Association. 6. Glycemic Targets: Standards of Medical Care in Diabetes-2019. Diabetes Care 2019;42:S61-70.

40 Bailey TS, Grunberger G, Bode BW, et al. American association of clinical endocrinologists and American College of endocrinology 2016 outpatient glucose monitoring consensus statement. Endocr Pract 2016;22:231-61.

41 Saragoni S, Perrone V, Buda S, et al. Analysis of clinical outcome and healthcare resource use in insulin treated diabetic patients based on self-monitoring of blood glucose levels. Int J Diabetol Vasc Dis Res 2017;5:183-8.

42 Bergenstal RM, Gavin JR, Global Consensus Conference on Glucose Monitoring Panel. The role of self-monitoring of blood glucose in the care of people with diabetes: report of a global consensus conference. Am J Med 2005;118:1-6.

43 Peel E, Parry O, Douglas M, et al. Blood glucose self-monitoring in non-insulin-treated type 2 diabetes: a qualitative study of patients' perspectives. Br J Gen Pract 2004;54:183-8.

44 McGill JB, Ahmann A. Continuous glucose monitoring with multiple daily insulin treatment: outcome studies. Diabetes Technol Ther 2017;19:S-3-S-12.

45 Abbott. Freestyle Libre pro system, 2019. Available: https://www. myfreestyle.com/freestyle-libre-pro-cgm-system [Accessed 26 Nov 2019].

46 Dexcom. Professional CGM, 2019. Available: https://provider. dexcom.com/products/professional-cgm [Accessed 26 Nov 2109].

47 Medtronic. Uncover more in less time. iPro ${ }^{\mathrm{TM}} 2$ professional continuous glucose monitoring (CGM). Available: https:// professional.medtronicdiabetes.com/ipro2-professional-cgm [Accessed 26 Nov 2019].

48 Reese P, Weispfenning R. Medtronic introduces Envision ${ }^{\mathrm{TM}}$ pro continuous glucose monitoring (CGM) system in Europe. Dublin, ireland: medtronic PLC, 2019. Available: http://newsroom.medtronic. com/node/30321/pdf [Accessed 3 Jan 2020].

49 Monnier L, Wojtusciszyn A, Colette C, et al. The contribution of glucose variability to asymptomatic hypoglycemia in persons with type 2 diabetes. Diabetes Technol Ther 2011;13:813-8.

50 Kovatchev B, Cobelli C. Glucose variability: timing, risk analysis, and relationship to hypoglycemia in diabetes. Diabetes Care 2016;39:502-10.

51 International Hypoglycaemia Study Group. Practice tools for patients \& care providers. Available: https://insgonline.com/ resources/tools-for-patients-and-care-providers/ [Accessed 24 Nov 2019].

52 Kloos C, Burghardt K, Müller UA, et al. Reduction of severe hypoglycaemia in people with type 2 diabetes after a structured inpatient intervention. Exp Clin Endocrinol Diabetes 2019. doi:10.1055/a-0983-1559 